\title{
Genetic parameters and prediction of genotypic values for root quality traits in cassava using REML/BLUP
}

\author{
E.J. Oliveira ${ }^{1}$, F.A. Santana ${ }^{2}$, L.A. Oliveira ${ }^{1}$ and V.S. Santos ${ }^{1}$ \\ ${ }^{1}$ Embrapa Mandioca e Fruticultura, Cruz das Almas, BA, Brasil \\ ${ }^{2}$ Universidade Federal do Recôncavo da Bahia, \\ Cruz das Almas, BA, Brasil \\ Corresponding author: E.J. Oliveira \\ E-mail: eder.oliveira@embrapa.br
}

Genet. Mol. Res. 13 (3): 6683-6700 (2014)

Received April 25, 2014

Accepted July 13, 2014

Published August 28, 2014

DOI http://dx.doi.org/10.4238/2014.August.28.13

\begin{abstract}
The aim of this study was to estimate the genetic parameters and predict the genotypic values of root quality traits in cassava (Manihot esculenta Crantz) using restricted maximum likelihood (REML) and best linear unbiased prediction (BLUP). A total of 471 cassava accessions were evaluated over two years of cultivation. The evaluated traits included amylose content (AML), root dry matter (DMC), cyanogenic compounds $(\mathrm{CyC})$, and starch yield (StYi). Estimates of the individual broad-sense heritability of AML were low $\left(h_{g}^{2}=0.07 \pm 0.02\right)$, medium for StYi and DMC, and high for CyC. The heritability of AML was substantially improved based on mean of accessions $\left(h_{m}^{2}=0.28\right)$, indicating that some strategies such as increasing the number of repetitions can be used to increase the selective efficiency. In general, the observed genotypic values were very close to the predicted average of the improved population, most likely due to the high accuracy $(>0.90)$, especially for DMC, CyC, and StYi. Gains via selection of the 30 best genotypes for each trait were 4.8 and $3.2 \%$ for an increase and decrease for AML, respectively, an
\end{abstract}


increase of 10.75 and $74.62 \%$ for DMC for StYi, respectively, and a decrease of $89.60 \%$ for $\mathrm{CyC}$ in relation to the overall mean of the genotypic values. Genotypic correlations between the quality traits of the cassava roots collected were generally favorable, although they were low in magnitude. The REML/BLUP method was adequate for estimating genetic parameters and predicting the genotypic values, making it useful for cassava breeding.

Key words: Breeding; Germplasm; Manihot esculenta Crantz; Selection; Genotypic value; Mixed models

\section{INTRODUCTION}

Cassava (Manihot esculenta Crantz) is the third most important source of calories in Africa, Asia, and Latin America (after rice and maize) and is characterized as a food security crop in these regions, where most of the cultivation occurs on marginal lands by small-scale farmers. According to the Food and Agriculture Organization of the United Nations (FAO, 2013), cassava has the potential to become the raw material base for a number of processed products that may increase the demand for cassava and contribute to agricultural transformation and economic growth in developing countries.

The genetic breeding of cassava has made significant progress, especially in increasing the crop yield potential through the development of new varieties (Kawano, 2003; Akinwale et al., 2010). Moreover, in addition to the yield and quality of the root, the variety component plays an important role in the diversification of the market due to cassava's starch-specific characteristics that allow for its broad use (Zhang et al., 2010). These characteristics include the contents of root starch, amylose (AML), cyanogenic compounds (CyC), soluble solids, and starch opacity.

Starch is a major component of cassava and has important food and industrial uses (Ceballos et al., 2007). In addition to its widespread application in the food industry, starch is also used in the textile, paper, metallurgy, pharmacy, and plastic industries (Aryee et al., 2006; Emmanuel et al., 2012). In general, cassava in Brazil is used primarily for the production of flour and starch. Thus, it is necessary for developing new varieties with differing starch characteristics that provide a competitive advantage over those that have been traditionally used in the production system.

Even at the international level, there are only a few cassava breeding programs; therefore, much knowledge is needed to optimize genetic gains for this crop. Although the main economic traits in cassava are quantitatively inherited and, therefore, highly influenced by environmental conditions, there have been few studies that have explored genetic variability in selecting the best genotypes for hybridization by using appropriate breeding methods that consider the inheritance of specific traits. In contrast, breeding progress is mainly determined by the size, nature, and inter-relationship of the genotypic and phenotypic variations of the various traits. Therefore, the separation of total variation into its inheritable and non-inheritable components is fundamental to define the progress and best strategies for selection. Accurate estimates of variance components and determinants for selection are of great importance in cassava breeding. These estimates can be obtained using optimal procedures of estimation/ 
prediction, thus enabling maximization of gains via selection (Furlani et al., 2005).

The prediction of breeding values for superior materials is the main problem in plant breeding because it requires the true values of the variance components; thus, it becomes necessary to use the BLUP (best linear unbiased prediction) and REML (restricted maximum likelihood) methods. BLUP assumes the knowledge of the variance component values, and estimates of these components by REML have been used. Both methods are associated with a linear mixed model. The use of mixed models in order to obtain predictors of breeding values (BLUP) is a method that has been used in several perennial (Resende and Barbosa, 2006; Hiraoka et al., 2011) and annual species (de Carvalho et al., 2008; Borges et al., 2010; Viana et al., 2010).

This study aimed to estimate the genetic parameters and select cassava genotypes using mixed model methodology. BLUP values and REML compounds were used to obtain the variance components for traits that are related to the yield and quality of cassava starch.

\section{MATERIAL AND METHODS}

\section{Plant material}

Four-hundred and seventy-one accessions from various ecosystems in Brazil, Colombia, Venezuela, and Nigeria were evaluated from the Cassava Germplasm Bank from Embrapa Mandioca e Fruticultura (Cruz das Almas, Brazil). This germplasm bank consists of landraces and improved varieties resulting from conventional breeding procedures such as crossing and selection and a selection of landraces with high-yield potential, as identified by farmers and research institutions.

\section{Experimental design}

Two field experiments were performed in 2011 and 2012 in the Experimental Fields Facilities at Embrapa Mandioca e Fruticultura, Cruz das Almas (Bahia, Brazil). In 2011, a randomized block design with three replications and 10 plants per plot was used. In 2012, an augmented block design with the number of accessions distributed homogeneously over 10 blocks with 10 plants per plot was used. Improved clones (9624-09 and 98150-06), landraces (Cigana and Eucalipto), and recommended varieties (BRS Aipim Brasil, BRS Dourada, BRS Verdinha, BRS Tapioqueira, BRS Caipira, and BRS Gema de Ovo) were used as the controls.

Planting was performed at the beginning of the rainy season (May to July) using 15$20-\mathrm{cm}$ stem cuttings in single rows. Spacing between rows and plants was 0.90 and $0.80 \mathrm{~m}$ between, respectively. All of the recommended cultivation practices for cassava were followed. The plants were harvested 11 months after planting.

\section{Sample preparation}

The root sampling was performed according to Rodriguez-Amaya and Kimura (2004). For each replicate, the roots of 10 plants from the plot were collected and mixed, and a random sub-sample of 5-6 roots was taken to represent the batch average. The roots selected were washed in tap water, cut into pieces, peeled (removing the bark), and cut into quarters. Two opposite sides of the roots were used for starch extraction. The other two opposing sides of the 
root quarters were chopped and crushed in a multiprocessor and mixer to form a homogeneous paste, which was used in the $\mathrm{CyC}$ and moisture analyses.

For starch extraction, cassava roots $(500 \mathrm{~g})$ were cut into pieces and ground in a blender, with non-cutting helix, helices thick per minute in a 1:1 ratio $(500 \mathrm{~g}$ cassava/500 $\mathrm{mL}$ cold water). Subsequently, this solution was filtered through a 150-mesh sieve covered with "voile" and then washed with cold water. Then, the recovered starch suspension was kept in a cold chamber at $5^{\circ} \mathrm{C}$ for $12 \mathrm{~h}$ to settle the starch. The supernatant was then discarded, and the starch was decanted, washed with approximately $20 \mathrm{~mL} \mathrm{95 \%} \mathrm{ethanol,} \mathrm{and} \mathrm{dried} \mathrm{in} \mathrm{an} \mathrm{oven}$ with forced air circulation at $40^{\circ} \mathrm{C}$ for $48 \mathrm{~h}$. The dried starch was ground to a fine-grained powder.

\section{Physicochemical analyses}

The determination of $\mathrm{CyC}$, especially cyanide, $\alpha$-hydroxynitrile lyase, and cyanogenic glycosides, present in the samples was performed according to Essers (1994) and consisted of extracting these compounds using a subsequent reaction with chloramine $\mathrm{T}$ and 1,3-isonicotinate-dimethyl barbiturate followed by spectrophotometric determination at $605 \mathrm{~nm}$. The linamarase enzyme, which was extracted from the bark of the cassava root, was used to release glycosidic cyanide according to Cooke (1979).

The root dry matter analysis (DMC) was performed in an oven with forced air circulation at $60^{\circ} \mathrm{C}$ for $48 \mathrm{~h}$ until a constant weight was obtained. The starch content was obtained by subtracting the DMC per $4.65 \%$, which is related to the ash, protein, lipid, and fiber contents. Therefore, the starch content was multiplied by the average fresh root yield, generating the starch yield (StYi) in $\mathrm{t} / \mathrm{ha}$.

The dried starch was analyzed in relation to the AML content according to ISO (1987). The starch sample gelatinized with sodium hydroxide and acidified with acetic acid was dispersed in $95 \%$ ethanol (pure for analysis). After the addition of the iodine solution, the blue color complex was quantified by spectrophotometry at $620 \mathrm{~nm}$ using a Biospectro model SP 220. For the AML analysis, only 445 accessions were analyzed according to the amount of available starch.

\section{Linear mixed model}

A combined analysis of different experimental designs was performed using models to incomplete the block designs. In this case, all of the blocks were set as random effects, while the design effects were treated as fixed by adjusting the experiments in a randomized complete block design and, at another level, for the experiments in an incomplete block design. In this case, the model set the block effects within each type of design.

The linear mixed model that was used to describe the data, which was according to Resende (2002a) as follows:

$$
y=X b+Z g+W p+e
$$

(Equation 1)

where $y$ is the data vector; $b$ is the vector of fixed effects associated with the overall average and block effect; $g$ is the vector of random genetic effects; $p$ is the vector of random effects of 
the plots; $e$ is the vector of random errors; and $X, Z$, and $W$ are incidence matrices that associate the unknown parameters $b, g$, and $p$, respectively, to the $y$ data vector.

The distributions and structures of means and variances were calculated by the following:

$$
\begin{aligned}
& y \mid b, V \sim N(X b, V) \\
& g \mid A, \sigma_{g}^{2} \sim N\left(0, A \sigma_{g}^{2}\right) \\
& p \mid \sigma_{p}^{2} \sim N\left(0, I \sigma_{p}^{2}\right) \\
& e \mid \sigma_{e}^{2} \sim N\left(0, I \sigma_{e}^{2}\right)
\end{aligned}
$$

The covariance between all of the random effect models was calculated by the following:

$$
\operatorname{Cov}\left(g, p^{\prime}\right)=0 ; \operatorname{Cov}\left(g, e^{\prime}\right)=0 \text { and } \operatorname{Cov}\left(p, e^{\prime}\right)=0 \quad \text { (Equation 3) }
$$

where

$$
E\left[\begin{array}{l}
y \\
g \\
p \\
e
\end{array}\right]=\left[\begin{array}{c}
X b \\
0 \\
0 \\
0
\end{array}\right]
$$

(Equation 4)

and

$$
\operatorname{Var}\left[\begin{array}{c}
y \\
g \\
p \\
e
\end{array}\right]=\left[\begin{array}{cccc}
V & Z G & W C & R \\
G Z^{\prime} & G & 0 & 0 \\
C W^{\prime} & 0 & C & 0 \\
R & 0 & 0 & R
\end{array}\right]
$$

where in

$$
G=A \hat{\sigma}_{g}^{2}, R=I \hat{\sigma}_{p}^{2}, C=I \hat{\sigma}_{e}^{2}
$$

and

$$
V=Z A \hat{\sigma}_{g}^{2} Z^{\prime}+W I \hat{\sigma}_{p}^{2} W+I \hat{\sigma}_{e}^{2}=Z G Z^{\prime}+W C W^{\prime}+R \quad \text { (Equation 7) }
$$


The mixed model methodology allows for the estimation of $b$ using a generalized least squares procedure, and $g$ and $p$ using BLUP. The system of linear equations [mixed model equations (MMEs)] that were used to obtain the solutions of the model were:

$$
\left[\begin{array}{l}
\hat{b} \\
\hat{g} \\
\hat{p}
\end{array}\right]=\left[\begin{array}{ccc}
X^{\prime} X & X^{\prime} Z & X^{\prime} W \\
Z^{\prime} X & Z^{\prime} Z+A^{-1} \lambda_{1} & Z^{\prime} W \\
W^{\prime} X & W^{\prime} Z & W^{\prime} W+I \lambda_{2}
\end{array}\right]^{-1}\left[\begin{array}{c}
X^{\prime} y \\
Z^{\prime} y \\
W^{\prime} y
\end{array}\right]
$$

where in

$$
\lambda_{1}=\frac{\sigma_{e}^{2}}{\sigma_{g}^{2}}=\frac{1-h^{2}-c^{2}}{h^{2}}
$$

and

$$
\lambda_{2}=\frac{\sigma_{e}^{2}}{\sigma_{p}^{2}}=\frac{1-h^{2}-c^{2}}{c^{2}}
$$

are the shrinkage factors of the random effects of the MMEs, in which $\sigma_{g}^{2}$ is the additive genetic variance, $\sigma_{p}^{2}$ is the variance of the effect of the plot, $\sigma_{e}^{2}$ is the residual variance (environment + non-additive), $A$ is the matrix of additive genetic correlation between individuals, and $I$ is the identity matrix.

REML estimates of the variance components were obtained using the expectation and maximization algorithm according to the following expressions:

$$
\begin{gathered}
\hat{\sigma}_{e}^{2}=\frac{y^{\prime} y-\hat{b}^{\prime} X^{\prime} y-\hat{g}^{\prime} Z^{\prime} y-\hat{p}^{\prime} W^{\prime} y}{N-r(x)} \\
\hat{\sigma}_{g}^{2}=\frac{\hat{g}^{\prime} A^{-1} \hat{g}+\hat{\sigma}_{e}^{2} \operatorname{tr}\left(A^{-1} C^{22}\right)}{q} \\
\hat{\sigma}_{p}^{2}=\frac{\hat{p}^{\prime} p+\hat{\sigma}_{e}^{2} \operatorname{tr} C^{33}}{s}
\end{gathered}
$$

where $C^{22}$ and $C^{33}$ were derived from: 


$$
C^{-1}=\left[\begin{array}{lll}
C_{11} & C_{12} & C_{13} \\
C_{21} & C_{22} & C_{23} \\
C_{31} & C_{32} & C_{33}
\end{array}\right]^{-1}=\left[\begin{array}{lll}
C^{11} & C^{12} & C^{13} \\
C^{21} & C^{22} & C^{23} \\
C^{31} & C^{32} & C^{33}
\end{array}\right]
$$

which is the generalized inverse of the coefficient MME matrix, $t r$ is the trace of a matrix, $r(x)$ is the rank of the $\mathrm{X}$ matrix, $N-r(x)$ is the number of degrees of freedom of the error, $q$ is the number individuals, $s$ is the number of plots, and $N$ is the total number of observations.

The coefficients of the individual broad-sense heritability of the block were estimated according to

$$
\hat{h}_{g}^{2}=\frac{\hat{\sigma}_{g}^{2}}{\hat{\sigma}_{g}^{2}+\hat{\sigma}_{p}^{2}+\hat{\sigma}_{e}^{2}}
$$

The standard deviation of the heritability coefficients was obtained from the information matrix of the MMEs. The other estimates of variances and genetic parameters were calculated by

$$
\hat{\sigma}_{f}^{2}=\hat{\sigma}_{g}^{2}+\hat{\sigma}_{p}^{2}+\hat{\sigma}_{e}^{2} \text { (phenotypic variance individual) }
$$

and

$$
\hat{h}_{m}^{2}=\frac{\hat{\sigma}_{g}^{2}}{\hat{\sigma}_{g}^{2}+\frac{\hat{\sigma}_{e}^{2}}{b}} \text { (heritability adjusted in average of accessions) (Equation 17) }
$$

where $b$ is the number of blocks. The accuracy of the selection of clones was given by

$$
A c=\sqrt{h_{m}^{2}}
$$

(Equation 18)

\section{Genetic gain}

The prediction estimates of REML/BLUP were performed using the Selegen software (Resende, 2002b). The genetic gain was calculated as the average of the breeding values of the selected individuals. The selection was performed using the breeding values of the 30 best genotypes for each trait.

The genotypic values of each clone were obtained by adding each genotypic effect to the overall mean. The genetic gain was equal to the average of the vectors of the predicted genetic effects for the selected accession. The overall mean plus the genetic gain resulted in the improved average population. The relative performance of each accession was determined using the relationship between the average of the improved population for each accession and the genetic value of the better accession, depending on the direction of selection.

\section{RESULTS AND DISCUSSION}

In the mixed model analysis using unbalanced data, the random effect models were 
tested by the likelihood ratio test, called the deviance analysis. The deviance values are shown in Table 1 and demonstrate the existence of significant differences among the accessions for all of the traits analyzed $(\mathrm{P}<0.05)$, indicating the existence of high levels of genetic variation among cassava accessions.

\begin{tabular}{|c|c|c|c|c|c|c|c|c|}
\hline \multirow[t]{2}{*}{ Effect } & \multicolumn{2}{|c|}{ AML } & \multicolumn{2}{|c|}{ DMC } & \multicolumn{2}{|c|}{$\mathrm{CyC}$} & \multicolumn{2}{|c|}{ StYi } \\
\hline & Deviance & LRT & Deviance & LRT & Deviance & LRT & Deviance & LRT \\
\hline $\begin{array}{l}\text { Genotypes } \\
\text { Complete model }\end{array}$ & $\begin{array}{l}3401.71 \\
3547.96\end{array}$ & $146.25^{* * *}$ & $\begin{array}{l}4852.32 \\
5342.04\end{array}$ & $489.72 * *$ & $\begin{array}{r}9504.33 \\
10403.49\end{array}$ & $899.16^{* *}$ & $\begin{array}{l}5561.5 \\
5973.3\end{array}$ & $411.80 * *$ \\
\hline
\end{tabular}

$\mathrm{AML}=$ amylose content; $\mathrm{DMC}=$ root dry matter; $\mathrm{CyC}=$ cyanogenic compounds; $\mathrm{StYi}=$ starch yield; LRT = likelibrood ratio test. $* *$ Significant at $5 \%$ of probability.

\section{Estimates of genetic parameters}

The prediction of breeding values was dependent on the knowledge of the genetic control of the trait and, in particular, the parameters of individual heritability and repeatability. Individual estimates of genetic parameters such as individual heritability are scarce in cassava and should therefore be investigated.

Variance components, heritability estimates, the coefficient of variation, and accuracy are presented in Table 2 . Although the AML trait was significantly different between the cassava accessions, the estimated individual broad-sense heritability was low $\left(h^{2}=0.07 \pm 0.02\right)$. However, the estimates of $h^{2}{ }_{g}$ were medium magnitude for StYi $(0.50 \pm 0.05)$ and DMC $(0.56$ $\pm 0.06)$, and high magnitude for $\mathrm{CyC}(0.74 \pm 0.06)$. The $h^{2}$ contains the additive and dominance variances between units of selection effectively used for the selection of better plants, especially considering that cassava genetic variance is transmitted by cloning.

The standard deviation for $h_{g}^{2}$ was always $<20 \%$ of the estimated heritability for all traits, except AML, ensuring a good prediction of breeding values according to Resende (2002a). Thus, there is good genetic control of the expression of these traits related to root quality; therefore, they show great potential for selection because the progress expected from selection depends directly on the heritability and the intensity of selection, which is inversely related to the phenotypic standard deviation (Dudley and Moll, 1969).

Individual heritability estimates were of median magnitudes for DMC and StYi, and of high magnitude for $\mathrm{CyC}$; however, there was high heritability for the average of the accessions ( $h_{m}^{2}$ of $0.80,0.85$, and 0.93 for StYi, DMC, and CyC, respectively). The main difference observed between $h_{g}^{2}$ and $h_{m}^{2}$ was attributed to lower phenotypic variation in the calculation of inheritance due to the reduction in residual variance and the absence of plot variance.

Different estimates of broad-sense heritability for DMC have been reported in the literature, such as 0.42 (Balyejusa et al., 2007), 0.56 (Akinwale et al., 2010), and 0.80 (Aina et al., 2007), which show that the results presented in this study are consistent with previous reports. However, according to Kawano et al. (1987), DMC is strongly influenced by the age of the plant, variety, and location and time of harvest. However, different values of heritability can be found for certain traits of the same species, mainly due to differences in the methods that are used for its determination, the genetic materials, the growth location, and the age of evaluation. 
Furthermore, Balyejusa et al. (2007) found estimates of broad-sense heritability for $\mathrm{CyC}$ to be of a lower magnitude than those observed in the present study (0.43), but the authors mention that the observed moderate heritability for $\mathrm{CyC}$ and $\mathrm{DMC}$ indicate that the phenotypic variations in these traits have genetic components that are important for QTL mapping. As a result, they identified two QTLs with additive effects for CyC as well as six QTLs, where one had an additive effect and the others had dominance/overdominance effects.

\begin{tabular}{|c|c|c|c|c|}
\hline Parameter & AML & DMC & CyC & $\overline{\text { StYi }}$ \\
\hline$\sigma_{g}^{2}$ & 0.37 & 8.46 & 438.97 & 11.27 \\
\hline$\sigma_{b}^{2}$ & 0.88 & 0.66 & 16.26 & 0.56 \\
\hline$\sigma_{e}^{2}$ & 3.85 & 6.07 & 139.34 & 10.93 \\
\hline$\sigma_{f}^{2}$ & 5.10 & 15.19 & 594.58 & 22.76 \\
\hline$h_{g}^{2}$ & $0.07 \pm 0.02$ & $0.56 \pm 0.06$ & $0.74 \pm 0.06$ & $0.50 \pm 0.05$ \\
\hline$c_{b}^{2}$ & 0.17 & 0.04 & 0.03 & 0.02 \\
\hline$h_{m}^{2}$ & 0.28 & 0.85 & 0.93 & 0.80 \\
\hline $\mathrm{Ac}$ & 0.53 & 0.92 & 0.96 & 0.90 \\
\hline $\mathrm{CVg}$ & 3.53 & 7.67 & 71.88 & 39.45 \\
\hline $\mathrm{CVe}$ & 11.33 & 6.50 & 40.50 & 38.84 \\
\hline $\mathrm{CVr}$ & 0.31 & 1.18 & 1.77 & 1.02 \\
\hline $\bar{X}_{f}$ & 17.32 & 37.93 & 29.15 & 8.51 \\
\hline
\end{tabular}

The importance of these results is that the high $h_{g}^{2}$ estimates for DMC, StYi, and CyC can contribute to rapid selection, even with the use of simple selection methods, such as phenotypic recurrent selection. In contrast, the lowest $h_{g}^{2}$ for AML indicates that the expression of this trait has great environmental influences and direct selection may be ineffective. Special attention should be given to AML, whose heritability was substantially improved when it was calculated using the average of the accessions $\left(h_{m}^{2}=0.28\right)($ Table 2). This procedure most likely attenuated the variation in repeated measurements and proved to be a method for increasing the efficiency of selection for this trait, which is of great importance in cassava breeding. Thus, it can be concluded that the results observed in this study indicate low heritability for AML, and strategies to increase the selection efficiency are required, such as an increase in the number of repetitions in the field.

Heritability estimates subsidizing the choice of the best breeding strategy reveal the potential for genetic progress with breeding and are essential for the prediction of breeding values. For example, da Costa et al. (2007) demonstrated using clonal tests in which 100 plants per clone led to accuracies $>90 \%$, regardless of broad-sense heritability, and that the appropriate number of teak (Tectona grandis L.f.) trees varies greatly depending on the individual 
broad-sense heritability. In contrast, for traits with a heritability of 0.20 , it is possible to obtain an accuracy of approximately $95 \%$ using 40 plants per clone and an accuracy of $90 \%$ using approximately 18 plants per clone. By using this knowledge for cassava breeding, it appears that fewer plants per genotype can be used for $\mathrm{DMC}, \mathrm{CyC}$, and StYi traits.

The REML allows for the estimation of the variance components in an unbalanced situation of experiments. This possibility is an interesting aspect in the context of cassava genetic breeding, whereas the implementation of trials with large numbers of accessions in balanced experiments is challenging because of the difficulties related to the low availability of propagation material for use in all repetitions and differential survival rates of genotypes, which is related to the quality of the stakes used for planting.

High values of the coefficient of determination due to the common environment of the plot $\left(\mathrm{c}_{\mathrm{b}}^{2}\right)$ indicated greater environmental variability between plots, as this parameter quantifies the variability within blocks. In our study, the $\mathrm{c}_{\mathrm{b}}{ }^{2}$ values ranged from 0.02 to 0.04 for $\mathrm{DMC}, \mathrm{CyC}$, and StYi, and 0.17 for AML (Table 2). According to Resende (2002a), the $\mathrm{c}_{\mathrm{b}}{ }^{2}$ value should be $<0.10$ for experiments of good quality.

Low genotypic coefficients of variation (CVg) were observed for AML and DMC (3.53 and $7.67 \%$, respectively). In contrast, $\mathrm{CVg}$ showed high genotypic variability in $\mathrm{CyC}$ and StYi (71.88 and $39.45 \%$, respectively) among cassava accessions. This genetic variability is a prerequisite for selecting the best accessions. For these same traits ( $\mathrm{CyC}$ and $\mathrm{StYi})$, the highest values of the coefficient of residual variation were observed (40.50 and $38.84 \%$ for $\mathrm{CyC}$ and StYi, respectively). However, the sole observation of this coefficient does not allow for a proper evaluation of experimental quality, which is necessary to consider the coefficient of relative variation $(\mathrm{CVr}=\mathrm{CVg} / \mathrm{CVe})$. Therefore, $\mathrm{CyC}$ was the trait with the highest $\mathrm{CVg}$ / $\mathrm{CVe}(\mathrm{CVr}=1.77)$, indicating a high possibility of selection gains for this trait. A similar situation was observed for DMC $(\mathrm{CVr}=1.18)$ and $\mathrm{StYi}(\mathrm{CVr}=1.02)$.

Experimental coefficients of variation $>50 \%$ were observed for the shoot weight and noncommercial root weight of the sweet potato (Ipomoea batatas L.) (Borges et al., 2010), as well as the shoot weight, yield per plant, and total yield per area of cassava (Aina et al., 2007). According to Borges et al. (2010), this magnitude of coefficient of variation, as well as other statistical parameters, is common when evaluating crops traits with underground structures, where environmental control is difficult. However, the maximum CVe observed in this experiment for cassava was $40.50 \%$ for CyC.

The accuracy values or correlation between the predicted and true genotypic values were $>0.90$ for $\mathrm{DMC}, \mathrm{CyC}$, and StYi. However, the accuracy for AML was 0.53 (Table 2). According to Resende and Duarte (2007), these accuracies are medium (0.53) for AML and very high (above 0.90) for DMC, CyC, and StYi.

High CVe values were observed for AML, which contributed to the achievement of low CVr estimates (0.31) and selective accuracy. However, this accuracy can be increased in future experiments using more adequate trials while maintaining the same size experiment but reducing the plot size and increasing the number of repetitions, as recommended by da Costa et al. (2007). These changes could increase the individual broad-sense heritability $\left(h_{g}^{2}\right)$ due to the smaller size and greater homogeneity of the block, which certainly contributed to the increase in selective accuracy. According to Resende and Duarte (2007), even with CVr values below unity, it is possible to achieve a selective accuracy $\geq 0.70$ if an adequate number of repetitions is used. 


\section{Prediction of genotypic values}

In the mixed model theory, assuming that the effects of the treatments are random, the multiple comparison tests that are usually implemented in the analysis of variance approach are not performed because these are derivatives of the supposition of treatment as fixed effects, and are applied and produce inferences about average phenotypic and not genotypic effects (Resende, 2002a). Considering the treatment effects to be random is essential for selecting and making inferences about the average values. Thus, the effects and predicted genotypic values, phenotypic values, genetic gains, and improved population mean and phenotypic values of the top 30 cassava accessions that were evaluated are presented in Tables 3, 4, and 5. For AML, the genotypic values ranged from 16.39 to $18.92 \%$, with an average of $17.32 \%$. Table 3 presents the 30 genotypes with the highest genotypic values for AML. The two improved varieties (BRS Dourada and BRS Gema de Ovo) showed higher genotypic AML values, while the improved clones were classified above the 133rd position.

Table 3. Rank (Rk) of cassava accessions and their genotypic values $(\mu+\mathrm{g})$, new averages (NA), and selective accuracies (Ac) for higher and lower amylose contents.

\begin{tabular}{|c|c|c|c|c|c|c|c|c|c|}
\hline \multicolumn{5}{|c|}{ Higher amylose content } & \multicolumn{5}{|c|}{ Lower amylose content } \\
\hline $\mathrm{Rk}$ & Accession & $\mu+\mathrm{g}$ & NA & $\mathrm{Ac}$ & $\mathrm{Rk}$ & Accession & $\mu+g$ & NA & Ac \\
\hline 1 & BRS Dourada & 18.92 & 18.92 & 0.76 & 445 & BGM0678 & 16.39 & 17.32 & 0.53 \\
\hline 2 & BRS Gema de Ovo & 18.26 & 18.59 & 0.76 & 444 & BGM0776 & 16.53 & 17.32 & 0.47 \\
\hline 3 & BGM1183 & 18.18 & 18.45 & 0.47 & 443 & BGM1186 & 16.56 & 17.32 & 0.47 \\
\hline 4 & BGM1184 & 18.15 & 18.38 & 0.53 & 442 & BGM0818 & 16.57 & 17.32 & 0.47 \\
\hline 5 & BGM0624 & 18.13 & 18.33 & 0.53 & 441 & BGM0834 & 16.59 & 17.32 & 0.47 \\
\hline 6 & BGM0631 & 18.09 & 18.29 & 0.53 & 440 & BGM1692 & 16.66 & 17.32 & 0.47 \\
\hline 7 & BGM0248 & 17.98 & 18.25 & 0.47 & 439 & BGM0847 & 16.66 & 17.33 & 0.53 \\
\hline 8 & BGM0367 & 17.97 & 18.21 & 0.53 & 438 & BGM0677 & 16.70 & 17.33 & 0.47 \\
\hline 9 & BGM1552 & 17.95 & 18.18 & 0.53 & 437 & BGM0184 & 16.71 & 17.33 & 0.53 \\
\hline 10 & BGM0885 & 17.95 & 18.16 & 0.53 & 436 & BGM0928 & 16.74 & 17.33 & 0.47 \\
\hline 11 & BGM0041 & 17.94 & 18.14 & 0.53 & 435 & BGM0956 & 16.76 & 17.33 & 0.40 \\
\hline 12 & BGM1333 & 17.94 & 18.12 & 0.53 & 434 & BGM0303 & 16.78 & 17.33 & 0.53 \\
\hline 13 & BGM1236 & 17.92 & 18.11 & 0.53 & 433 & Cigana & 16.80 & 17.33 & 0.76 \\
\hline 14 & BGM0540 & 17.90 & 18.09 & 0.53 & 432 & BGM0800 & 16.81 & 17.34 & 0.53 \\
\hline 15 & BGM0360 & 17.86 & 18.08 & 0.53 & 431 & BGM0089 & 16.81 & 17.34 & 0.47 \\
\hline 16 & BGM0331 & 17.86 & 18.06 & 0.53 & 430 & BGM1123 & 16.81 & 17.34 & 0.53 \\
\hline 17 & BGM1100 & 17.85 & 18.05 & 0.47 & 429 & BGM1085 & 16.82 & 17.34 & 0.47 \\
\hline 18 & BGM0103 & 17.84 & 18.04 & 0.53 & 428 & BGM0273 & 16.83 & 17.34 & 0.53 \\
\hline 19 & BGM1190 & 17.81 & 18.03 & 0.53 & 427 & BGM0499 & 16.84 & 17.34 & 0.47 \\
\hline 20 & BGM0204 & 17.80 & 18.02 & 0.47 & 426 & BGM0341 & 16.85 & 17.34 & 0.53 \\
\hline 21 & BGM0823 & 17.79 & 18.00 & 0.53 & 425 & 9624-09 & 16.85 & 17.34 & 0.76 \\
\hline 22 & BGM0901 & 17.79 & 17.99 & 0.53 & 424 & BGM0455 & 16.86 & 17.34 & 0.47 \\
\hline 23 & BGM0057 & 17.79 & 17.99 & 0.53 & 423 & BGM0434 & 16.87 & 17.35 & 0.47 \\
\hline 24 & BGM0166 & 17.78 & 17.98 & 0.53 & 422 & BGM0390 & 16.88 & 17.35 & 0.53 \\
\hline 25 & BGM0547 & 17.78 & 17.97 & 0.53 & 421 & BGM0093 & 16.89 & 17.35 & 0.53 \\
\hline 26 & BGM1491 & 17.78 & 17.96 & 0.40 & 420 & BGM1361 & 16.89 & 17.35 & 0.53 \\
\hline 27 & BGM1623 & 17.78 & 17.96 & 0.53 & 419 & BGM0649 & 16.90 & 17.35 & 0.47 \\
\hline 28 & BGM1495 & 17.77 & 17.95 & 0.47 & 418 & BGM0767 & 16.90 & 17.35 & 0.53 \\
\hline 29 & BGM1124 & 17.76 & 17.94 & 0.47 & 417 & BGM1440 & 16.92 & 17.35 & 0.47 \\
\hline 30 & BGM0295 & 17.75 & 17.94 & 0.40 & 416 & 98150-06 & 16.94 & 17.35 & 0.47 \\
\hline
\end{tabular}

The breeder should implement strategies to predict the individual genetic value and plan how to use individuals with the highest breeding values. In cassava, it is possible to operate using two basic strategies. The first one involves the generation of populations formed by descendants of individuals selected, where the selection will act by altering the allele frequencies in the loci controlling the traits under selection to alter the genotypic mean of the popula- 
tion in the desired direction. In this case, for segregating populations derived from the crossing of superior genotypes, it is important to estimate the additive genetic values, considering that only these additive effects are transmitted to offspring via sexual reproduction. In the second strategy, the goal is to select individuals with the highest predicted values, as it is possible to use clonal propagation. In this situation, it is interesting to estimate genotypic values that capitalize the additive and dominance effects, which are fully transmitted to the next generation through vegetative propagation.

Table 4. Rank (Rk) of cassava accessions and their genotypic values $(\mu+\mathrm{g})$, new averages (NA), and selective accuracies $(\mathrm{Ac})$ for dry matter content $(\mathrm{DMC})$ and cyanogenic compounds $(\mathrm{CyC})$.

\begin{tabular}{|c|c|c|c|c|c|c|c|c|c|}
\hline \multicolumn{5}{|c|}{ DMC } & \multicolumn{5}{|c|}{$\mathrm{CyC}$} \\
\hline $\mathrm{Rk}$ & Accession & $\mu+\mathrm{g}$ & NA & $\mathrm{Ac}$ & $\mathrm{Rk}$ & Accession & $\mu+g$ & NA & Ac \\
\hline 1 & BGM1202 & 43.46 & 43.46 & 0.92 & 471 & BGM0341 & 0.00 & 29.15 & 0.96 \\
\hline 2 & BGM0443 & 43.17 & 43.32 & 0.92 & 470 & BGM1195 & 0.00 & 29.22 & 0.95 \\
\hline 3 & BGM0027 & 43.08 & 43.24 & 0.92 & 469 & BGM0376 & 0.00 & 29.28 & 0.96 \\
\hline 4 & BGM0656 & 43.02 & 43.18 & 0.86 & 468 & BGM0375 & 0.00 & 29.34 & 0.95 \\
\hline 5 & BGM2043 & 42.44 & 43.03 & 0.92 & 467 & BGM0868 & 0.17 & 29.40 & 0.96 \\
\hline 6 & BGM0425 & 42.38 & 42.93 & 0.92 & 466 & BGM1491 & 0.93 & 29.47 & 0.93 \\
\hline 7 & BGM0144 & 42.36 & 42.85 & 0.92 & 465 & BGM0656 & 0.98 & 29.53 & 0.93 \\
\hline 8 & BGM1116 & 42.33 & 42.78 & 0.92 & 464 & BGM1526 & 1.00 & 29.59 & 0.93 \\
\hline 9 & BGM1311 & 42.22 & 42.72 & 0.92 & 463 & BGM0367 & 1.00 & 29.65 & 0.96 \\
\hline 10 & BGM0341 & 42.17 & 42.66 & 0.92 & 462 & BGM1100 & 1.29 & 29.71 & 0.95 \\
\hline 11 & BGM0542 & 42.14 & 42.62 & 0.92 & 461 & BGM0433 & 1.34 & 29.77 & 0.93 \\
\hline 12 & BGM0940 & 42.03 & 42.57 & 0.90 & 460 & BGM0800 & 1.75 & 29.84 & 0.95 \\
\hline 13 & BGM0544 & 42.02 & 42.52 & 0.92 & 459 & BGM0041 & 2.02 & 29.90 & 0.96 \\
\hline 14 & BGM0620 & 41.98 & 42.49 & 0.90 & 458 & BGM0254 & 2.92 & 29.96 & 0.96 \\
\hline 15 & BGM1450 & 41.91 & 42.45 & 0.76 & 457 & BGM0330 & 3.14 & 30.02 & 0.96 \\
\hline 16 & BGM1171 & 41.85 & 42.41 & 0.92 & 456 & BGM0542 & 3.45 & 30.08 & 0.96 \\
\hline 17 & BGM0783 & 41.80 & 42.37 & 0.90 & 455 & BGM0443 & 3.52 & 30.13 & 0.96 \\
\hline 18 & BGM1692 & 41.77 & 42.34 & 0.90 & 454 & BGM0624 & 3.65 & 30.19 & 0.96 \\
\hline 19 & BGM0845 & 41.75 & 42.31 & 0.92 & 453 & BGM0631 & 3.85 & 30.25 & 0.96 \\
\hline 20 & BGM1495 & 41.74 & 42.28 & 0.92 & 452 & BGM0276 & 3.99 & 30.31 & 0.95 \\
\hline 21 & BGM0818 & 41.61 & 42.25 & 0.90 & 451 & BGM0149 & 4.10 & 30.37 & 0.96 \\
\hline 22 & BGM0143 & 41.60 & 42.22 & 0.76 & 450 & BGM0642 & 5.00 & 30.43 & 0.96 \\
\hline 23 & BGM1956 & 41.51 & 42.19 & 0.76 & 449 & BGM0501 & 5.24 & 30.48 & 0.96 \\
\hline 24 & BGM0196 & 41.51 & 42.16 & 0.90 & 448 & BGM0434 & 5.30 & 30.54 & 0.95 \\
\hline 25 & BGM0587 & 41.49 & 42.13 & 0.90 & 447 & BGM0307 & 5.39 & 30.60 & 0.95 \\
\hline 26 & BGM0455 & 41.49 & 42.11 & 0.90 & 446 & BGM0464 & 5.87 & 30.65 & 0.93 \\
\hline 27 & BGM0428 & 41.43 & 42.08 & 0.92 & 445 & BGM0211 & 5.97 & 30.71 & 0.96 \\
\hline 28 & BGM0800 & 41.38 & 42.06 & 0.92 & 444 & BGM1517 & 6.15 & 30.76 & 0.96 \\
\hline 29 & BGM1362 & 41.38 & 42.04 & 0.76 & 443 & BGM0908 & 6.24 & 30.82 & 0.95 \\
\hline 30 & BGM0821 & 41.30 & 42.01 & 0.92 & 442 & BGM0356 & 6.69 & 30.88 & 0.96 \\
\hline
\end{tabular}

Considering the generation of segregating populations from crosses of the 30 best genotypes, the new average of the population would be $18.14 \%$ for AML, which corresponds to a gain of 4.8 and $2.1 \%$ in comparison to the averages of the experiment and controls, respectively. In contrast, if the strategy uses the genotypes with higher average predicted values for commercial use, the varieties BRS Dourada and BRS Gema de Ovo would be optimal for obtaining higher AML contents (Table 3). Other literature reports AML phenotypic values ranging from 17.9 to $23.6 \%$ (Defloor et al., 1998), 18.0 to 25.0\% (Moorthy, 2004), 13.6 to $23.8 \%$ (Rickard et al., 1991), and 0.0\% AML, known as the waxy variety (Ceballos et al., 2007). In the last case, the functional properties of waxy starch are different from those of normal starches, including a high viscosity, high swelling index, and low solubility. Other products such as glucose, fructose syrups, and other beverages, are being developed, depending on the starch and amylose contents from cassava varieties (Aryee et al., 2006). 
Table 5. Rank (Rk) of cassava accessions and their genotypic values $(\mu+\mathrm{g})$, new averages (NA), and selective accuracies (Ac) for starch yield (StYi).

\begin{tabular}{rccccccccc}
\hline Rk & Accession & $\mu+\mathrm{g}$ & NA & Ac & Rk & Accession & $\mu+g$ & NA & Ac \\
\hline 1 & BGM1626 & 19.05 & 19.05 & 0.90 & 16 & BGM0279 & 14.25 & 15.66 \\
2 & BGM0120 & 17.93 & 18.49 & 0.89 & 17 & BGM2044 & 14.11 & 15.57 & 0.89 \\
3 & BGM2020 & 16.71 & 17.90 & 0.71 & 18 & BGM1598 & 14.09 & 15.48 \\
4 & BGM1200 & 16.47 & 17.54 & 0.89 & 19 & BGM0165 & 14.07 & 15.41 & 0.90 \\
5 & BRS Caipira & 15.88 & 17.21 & 0.97 & 20 & BGM0937 & 14.05 & 15.34 & 0.87 \\
6 & Filha da Preta & 15.81 & 16.97 & 0.71 & 21 & 9624-09 & 14.03 & 15.28 \\
7 & BGM0892 & 15.48 & 16.76 & 0.89 & 22 & BGM0011 & 14.02 & 15.22 \\
8 & BGM0163 & 15.39 & 16.59 & 0.89 & 23 & BGM0337 & 14.01 & 15.17 \\
9 & BGM0425 & 15.25 & 16.44 & 0.89 & 24 & BGM0049 & 13.98 & 15.12 & 0.87 \\
10 & BGM0823 & 15.09 & 16.30 & 0.89 & 25 & BGM1165 & 13.94 & 15.07 \\
11 & BGM0010 & 14.92 & 16.18 & 0.89 & 26 & BGM0303 & 13.88 & 15.03 & 0.89 \\
12 & BGM1085 & 14.86 & 16.07 & 0.87 & 27 & BGM0598 & 13.80 & 14.98 & 0.89 \\
13 & BGM0394 & 14.61 & 15.96 & 0.89 & 28 & BGM0332 & 13.79 & 14.94 & 0.89 \\
14 & BGM0847 & 14.47 & 15.85 & 0.89 & 29 & BGM0276 & 13.78 & 14.90 & 0.87 \\
15 & BGM1282 & 14.35 & 15.75 & 0.87 & 30 & BGM0039 & 13.62 & 14.86 & 0.89 \\
\hline
\end{tabular}

The reduction in AML is an interesting attribute for the starch industry; thus, we selected 30 accessions with lower AML contents (Table 3). In this case, the genotypic values ranged from 16.39 to $16.94 \%$, with an average of $16.76 \%$. In addition to 27 other germplasm accessions, two clones (9624-09 and 98150-06) and a local variety (Cigana) were selected based on this criterion. The genetic gain (reduced amylose) with the selection of these genotypes was 3.2 and $5.6 \%$ in comparison to the overall average of the experiments and controls, respectively. The two accessions with the lowest AMLs were BGM0678 and BGM0776, with predicted averages of 16.39 and $16.53 \%$, respectively (Table 3 ).

Among the various prediction methods, BLUP has the ability to maximize selective accuracy (Resende, 2002a). However, the selective accuracies in both accessions with high and low AMLs were relatively low (ranging from 0.40 to 0.76 ), which compromises the accurate selection of the best genotypes for an increase or decrease in this trait based on the current data. However, as mentioned earlier, the accuracy of this trait can be improved by reducing the plot size and increasing the number of experimental repetitions.

For DMC, genotypic values for all cassava accessions ranged from 24.93 to $43.46 \%$. Low variation in the phenotypic data was observed by Raji et al. (2007) (33.2-39.2\%) and can be explained by the lower number of cassava accessions evaluated, thus restricting the genetic variability to be sampled. In relation to the DMC trait, the 30 top clones showed genotypic values ranging from 41.30 to $43.46 \%$, with an average of $42.01 \%$. The genetic gain from selecting those 30 accessions was $10.75 \%$ and $5.5 \%$ in comparison to the overall average of the experiments and controls, respectively. The selective accuracy for this trait was quite high (0.76-0.92) (Table 4), which ensures greater precision in selecting the optimal cassava accessions for expressing this trait. Moreover, Akinwale et al. (2010) reported median estimates of genetic heritability and low gains $(8.7 \%)$ for $\mathrm{DMC}$, which may be due to lower variability in the varieties that were examined by these authors.

The DMC increase is important because, according to Rickard et al. (1991), approximately $74-85 \%$ of the dry root weight is starch. Therefore, the DMC directly affects the amount of starch that is extracted from the cassava roots. In addition, the starch producers in Brazil generally pay different prices for fresh roots depending on the DMC.

The predicted values for $\mathrm{CyC}$ showed a wide variation, ranging from 0.00 to 115.53 
$\mathrm{mg} / \mathrm{kg}$ (considering all genotypes). When evaluating 11 cassava varieties from Nigeria, Raji et al. (2007) observed phenotypic variation similar to that of CyC $(40.0 \mathrm{mg} / \mathrm{kg}$ from landrace Isunikankiyan to $128.6 \mathrm{mg} / \mathrm{kg}$ from cultivar TMS30572).

According to Jansz and Uluwaduge (1997), based on the contents of the CyCs, the cassava roots can be classified into three classes, including low toxicity or sweet cassava $(<50$ $\mathrm{mg} / \mathrm{kg}$ ), medium toxicity (between 50 and $100 \mathrm{mg} / \mathrm{kg}$ ), and high toxicity or bitter cassava $(>100 \mathrm{mg} / \mathrm{kg})$. In general, the sweet cassava market demands varieties with good cooking quality and low levels of CyCs (linamarin and lotaustralin), which produce hydrogen cyanide. The identification of cassava varieties with low in natura $\mathrm{CyC}$ levels in the roots is necessary to reduce the risk of poisoning consumers (Jansz and Uluwaduge, 1997). Moreover, the processing steps of the roots (fermentation, pressing, washing, drying, and exposure to high temperatures) used in the manufacture of flour and starch greatly reduce the CyC levels (Piyachomkwan et al., 2005). Thus, considering that most of the production of cassava roots is destined for industrial flour and starch, the $\mathrm{CyC}$ content is not a major trait in the selection of varieties for this type of market, wherein high productivity and starch content are the most valued traits.

The wide variation in the $\mathrm{CyC}$ content found in this study certainly contributed to a suitable choice of the best genotypes for diverse uses, with a special focus on the fresh market (in natura). Thus, the selection of the 30 best clones with lower $\mathrm{CyC}$ values showed genotypic variation from 0.00 to $6.69 \%$, averaging $3.03 \%$. The genetic gain (in the sense of reduction) to select these 30 accessions was 89.60 and $88.07 \%$ in comparison to the overall average of the experiments and controls, respectively. Furthermore, the selective accuracy of this trait was also quite high (0.93-0.96) (Table 4).

Mainly for the flour and starch industries, the amount of starch is crucial in choosing which variety to use in a large-scale production system where it is necessary to maximize the efficiency of production per hectare. In this sense, it is important to consider the productive potential of the variety and its starch content, which, in the current study, was StYi (Table 5). Similar to other traits, the predicted genotypic values were quite variable among the accessions (3.24-19.05 t/ha), making this population a good choice for selecting the best individuals and use as a parental population to generate segregating populations. In these instances, there would be accurate selection of these accessions, considering that the magnitude of selective accuracy ranged from high (0.71-0.89 in 25 accessions) to very high ( $>0.90$ in five accessions) (Table 5).

For StYi, the top 30 clones showed genotypic values ranging from 13.62 to $19.05 \%$, with an average of $8.51 \%$. The genetic gains by selecting those accessions were 74.62 and $49.95 \%$ in comparison to the overall average of the experiments and controls, respectively (Table 5). Among the controls, one clone (9624-09), one recommended variety (BRS Caipira), and one local variety (Filha da Preta) stood out as the most promising accessions for StYi.

Generally, for all of the root quality traits of cassava, we observed that the genotypic values $(\mu+\mathrm{g})$ were very close to the new average because REML/BLUP was the best estimator of genotypic values (Resende, 2002a; Klapště et al., 2007; de Carvalho et al., 2008; Viana et al., 2012). Furthermore, this methodology has the following main advantages, including that it (a) has applications in experiments with unbalanced data, (b) does not require data from rigid experimental structures, (c) allows for the simultaneous use of a large amount of information from various experiments, thus generating more accurate estimates, and (d) corrects 
the data for environmental effects accurately and, without bias, predicts the genotypic values, thus maximizing the selective genetic gain (Resende, 2002a).

One point of concern is the ability of breeders to select the best genotypes to be used for crossing to generate segregating populations or per se use in the production system. If the goal of the program is to promote the recombination of the best genotypes, then selection should be made based on the additive genetic values of individuals. In contrast, if the goal is to clone the genotype, selection should be made using genotypic values, which are necessary to obtain non-additive variance. In the latter case, making inferences about the genetic materials in field experiments in order to rank those candidates to be released as cultivars is no easy task, as these inferences must be based on true genotypic values. For example, inferring genotypes at any stage of a breeding program should be based on the genetic and not phenotypic means because the genotypic averages indicate future averages when the cultivars are planted in commercial fields (Borges et al., 2010). In this context, the use of improved prediction methods such as BLUP provides higher selection accuracy and, thus, dramatically increases accuracy in the evaluation and selection of superior genotypes.

The high level of diversity observed for root quality traits can be used to aid in the development of cassava segregating populations with specific traits for use in food, feed, or for industrial purposes. This study also revealed new sources for low $\mathrm{CyCs}$ and high starch productivity in addition to providing useful information regarding the inheritance of these traits to better plan strategies for selection and recombination in cassava breeding programs.

\section{Genetic correlations}

The genetic correlation ( $\mathrm{rg}$ ) for AML was greater in magnitude than that of the phenotypic correlation. For other combinations, the phenotypic correlations were greater (Table 6). The phenotypic correlations may be smaller than the genetic correlations when associated with low and positive environmental correlations and when controlled by similar genes.

$\begin{aligned} & \text { Table 6. Estimates of genetic (above the diagonal) and phenotypic (below the diagonal) correlation coefficients } \\
& \text { in cassava accessions for amylose content (AML), dry matter content (DMC), cyanogenic compounds (CyC), } \\
& \text { and starch yield (StYi). }\end{aligned}$
\begin{tabular}{lcccr}
\hline Traits & AML & DMC & CyC & StYi \\
\hline AML & - & 0.25 & -0.10 & 0.46 \\
DMC & 0.25 & - & 0.18 & 0.36 \\
CyC & 0.05 & 0.29 & - & 0.01 \\
StYi & 0.26 & 0.42 & 0.05 & - \\
\hline
\end{tabular}

The rg among the root quality traits of cassava that was obtained in this study was generally favorable, although low in magnitude. Favorable rg between AML x DMC (0.25), AML x StYi (0.46), and DMC x StYi (0.36) (Table 6) indicates that the increase in the DMC may elevate the amylose content and starch yields of cassava roots. On the other hand, no significant rg was observed for CyC. Moreover, Dixon et al. (1994) reported that the values of the phenotypic correlation between $\mathrm{DMC}$ and $\mathrm{CyC}$ are negatively correlated, with variation 
ranging from -0.73 to -0.55 , depending on the genotype or location.

Although genetic correlations can quantify the possibility of indirect gains by selection on correlated traits, especially for those with low heritability, we found that traits of the cassava roots cannot be efficiently and indirectly selected using other correlations because of the low genetic correlations.

\section{Perspectives for use in cassava breeding}

In cassava, variation plays an important role in producing diverse foods due to the inherent characteristics that vary with genotype (Ceballos et al., 2007; Zhang et al., 2010). Such traits include amylose and starch contents, CyCs, carotenoids, and others. These traits are used in breeding programs without neglecting yield, earliness, resistance to pests and diseases, or adaptability to different production regions. Thus, improvements in new methodologies for genetic analysis aimed at increasing yield levels and the quality of cassava starch is increasingly important for ensuring the competitiveness and sustainability of cassava in Brazil. According to Balyejusa et al. (2007), until recently, cassava breeding programs conducted their work without much knowledge of the genetic architecture of the main traits under selection, which certainly tends to reduce the efficiency of breeding and selection gains.

In addition, there are other important problems, such as the long breeding cycle of cassava, which lasts at least five years when calculating a year for each of the following steps: evaluation of seedlings; and the clonal, preliminary, and advanced trials and crossings of the best individuals. This long period is necessary because of the low multiplication rates of cassava (1:6, on average), which means there would be enough propagation material to repeat trials only in the later stages. Therefore, the high cost of extensive and time-consuming progeny evaluations under field conditions requires the accurate estimation of variance components for the prediction of breeding values and maximizing the accuracy of selection in cassava breeding programs.

Throughout this process, selecting the wrong genotypes for crossing or those used in regional trials can pose a serious problem. In this context, the individual BLUP predicts the breeding values of the random effects of the statistical model associated with phenotypic observations, fitting the data to the fixed effects and the unequal amount of information in the plots by mixed model methodology (Resende, 2002a). Thus, it is expected that the use of improved prediction methods such as BLUP provides greater accuracy in selection and drastically reduces the period of genotypic evaluation, as the estimation of genotypic values can be initiated during the seedling stage using familial information.

A seedling stage produces thousands of unique heterozygote individuals originating from crosses between parents selected. Thus, the selection performed at this stage for traits with low individual heritability, such as AML, would not be indicated. However, the selection would be more effective if based on familial information in which most of the phenotypic variation can be explained by genetic components. In contrast, according to Latter (1964), when the heritability exceeds 0.50 , selection based on individuals tends to be higher than that based on families. Therefore, the $h_{g}^{2}$ estimates for StYi, DMC, and CyC when applied individually during the early stages of cassava breeding may contribute to the identification of promising new families for these traits and yield a greater number of genotypes for potential use as new cultivars. 


\section{ACKNOWLEDGMENTS}

The authors thank Fundação de Amparo à Pesquisa do Estado da Bahia (Fapesb) and Conselho Nacional de Desenvolvimento Científico e Tecnológico (CNPq) for financial assistance and scholarship support.

\section{REFERENCES}

Aina OO, Dixon AGO and Akinrinde EA (2007). Genetic variability in cassava as it influences storage root yield in Nigeria. J. Biol. Sci. 7: 765-770.

Akinwale MG, Akinyele BO, Dixon AGO and Odiyi AC (2010). Genetic variability among forty-three cassava genotypes in three agro-ecological zones of Nigeria. J. Plant Breed. Crop Sci. 2: 104-109.

Aryee FNA, Oduro I, Ellis WO and Afuakwa JJ (2006). The physicochemical properties of flour samples from the roots of 31 varieties of cassava. Food Control 17: 916-922.

Balyejusa KE, Rönnberg-Wästljung AC, Egwang T, Gullberg U, et al. (2007). Quantitative trait loci controlling cyanogenic glucoside and dry matter content in cassava (Manihot esculenta Crantz) roots. Hereditas 144: 129-136.

Borges V, Ferreira PV, Soares L, Santos GM, et al. (2010). Seleção de clones de batata-doce pelo procedimento REML/ BLUP. Acta Sci. Agron. 32: 643-649.

Ceballos H, Sanchez T, Morante N, Fregene M, et al. (2007). Discovery of an amylose-free starch mutant in cassava (Manihot esculenta Crantz). J. Agric. Food Chem. 55: 7469-7476.

Cooke RD (1979). Enzymatic Assay for Determining the Cyanide Content of Cassava and Cassava Products. Centro International de Agricultura Tropical (CIAT), Cali.

da Costa RB, Deon M and de Resende V (2007). Experimentação e seleção no melhoramento genético da teca (Tectona grandis L.f.). Flor. Amb. 14: 76-92.

de Carvalho ADF, Fritsche Neto R and Geraldi IO (2008). Estimation and prediction of parameters and breeding values in soybean using REML/BLUP and Least Squares. Crop Breed. Appl. Biotechnol. 8: 219-224.

Defloor I, Dehing I and Delcour JA (1998). Physico-chemical properties of cassava starch. Starch 8: 58-64.

Dixon AGO, Asiedu R and Bokanga M (1994). Breeding of cassava for low cyanogenic potential: problems, progress and perspectives. Acta Hort. 375: 153-161.

Dudley JW and Moll RH (1969). Interpretation and use of estimates of heritability and genetic variances in plant breeding. Crop Sci. 9: 257-262.

Emmanuel OA, Clement A, Agnes SB, Chiwona-Karltun L, et al. (2012). Chemical composition and cyanogenic potential of traditional and high yielding CMD resistant cassava (Manihot esculenta Crantz) varieties. Int. Food. Res. J. 19: 175-181.

Essers AJ (1994). Further improving the enzymic assay for cyanogens in cassava products. Acta Hortic. 375: 97-104.

FAO (2013). Agriculture: Cassava. Available at [http://www.fao.org/ag/agp/agpc/gcds/]. Accessed July 14, 2013.

Furlani RCM, Moraes MLT, Resende MDV and Furlani Junior E (2005). Estimation of variance components and prediction of breeding values in rubber tree breeding using the REML/BLUP procedure. Genet. Mol. Biol. 28: 271-276.

Hiraoka Y, Kuramoto N, Ohira M and Okamura M (2011). Estimation of genetic data and breeding values of traits related to wax production in Rhus succedanea L. clones using the REML/BLUP method. J. For. Res. 16: 509-517.

ISO International Organization for Standardization (1987). Norme ISO 6647 (F). Riz-Determination de La Teneur en Amylose, Switzerland.

Jansz ER and Uluwaduge DI (1997). Biochemical aspects of cassava (Manihot esculenta Crantz) with special emphasis on cyanogenic glucosides. J. Natn. Sci. Foundation Sri Lanka 25: 1-24.

Kawano K (2003). Thirty years of cassava breeding for productivity-biological and social factors for success. Crop Sci. 43: 1325-1335.

Kawano K, Fukuda WMG and Cenpukdee U (1987). Genetic and environmental effects on dry matter content of cassava root. Crop Sci. 27: 69-74.

Klapště J, Lstibůrek M and Kobliha J (2007). Initial evaluation of half-sib progenies of Norway spruce using the best linear unbiased prediction. J. For. Sci. 53: 41-46.

Latter BDH (1964). Selection Methods in the Breeding of Cross-Fertlized Pasture Species. In: Grasses and Grasslands (Barnard C, ed.). MacMillan, London, 168-181.

Moorthy SN (2004). Tropical Sources of Starch. In: Starch in Food (Eliasson AC, ed.). CRC Press, Boca Raton, 321-359.

Piyachomkwan K, Wanlapatit S, Chotineeranat S and Sriroth K (2005). Transformation and balance of cyanogenic compounds in the cassava starch manufacturing process. Starch 57: 71-78.

Genetics and Molecular Research 13 (3): 6683-6700 (2014)

CFUNPEC-RP www.funpecrp.com.br 
Raji AA, Ladeinde TAO and Dixon AGO (2007). Agronomic traits and tuber quality attributes of farmer grown cassava landraces in Nigeria. J. Agric. Trop. 45: 9-13.

Resende MDV (2002a). Genética Biométrica e Estatística no Melhoramento de Plantas Perenes. Embrapa Informação Tecnológica, Brasília.

Resende MDV (2002b). Software Selegen-Reml/Blup. Embrapa Florestas, Colombo.

Resende MDV and Barbosa MHP (2006). Selection via simulated BLUP based on family genotypic effects in sugarcane. Pesq. Agropec. Bras. 41: 421-429.

Resende MDV and Duarte JB (2007). Precisão e controle da qualidade de experimentos para avaliação de cultivares. Pesq. Agropec. Trop. 37: 182-194.

Rickard JE, Asaoka M and Blanshard JMV (1991). Review of the physicochemical properties of cassava starch. Trop. Sci. 31: 189-207.

Rodriguez-Amaya DB and Kimura M (2004). HarvestPlus Handbook for Carotenoid Analysis. IFPRI, Washington.

Viana JMS, Sobreira FM, Resende MDV and Faria VR (2010). Multi-trait BLUP in half-sib selection of annual crops. Plant Breed. 129: 599-604.

Viana JMS, Faria VR, Silva FF and Resende MDV (2012). Combined selection of progeny in crop breeding using best linear unbiased prediction. Can. J. Plant Sci. 92: 553-562.

Zhang P, Wang WQ, Zhang GL, Kaminek M, et al. (2010). Senescence-inducible expression of isopentenyl transferase extends leaf life, increases drought stress resistance and alters cytokinin metabolism in cassava. J. Integr. Plant Biol. 52: 653-669. 